\title{
Symmetric monochromatic subsets in colorings of the Lobachevsky plane
}

\author{
Taras Banakh ${ }^{1}$, Artem Dudko ${ }^{2}$, and Dušan Repovš $\check{3}^{3}$ \\ ${ }^{1}$ Department of Mathematics, Lviv National University, Lviv, Ukraine, \\ and Instytut Matematyki, Uniwersytet Humanistyczno-Przyrodniczy J.Kochanowskiego, Kielce, Poland \\ E-mail: tbanakh@yahoo.com \\ ${ }^{2}$ University of Toronto, 40 St. George Street Toronto, Ontario, Canada M5S 2E4 \\ E-mail: artemdudko@rambler.ru, artem.dudko@utoronto.ca \\ ${ }^{3}$ Faculty of Mathematics and Physics, and Faculty of Education, University of Ljubljana, Jadranska 19, Ljubljana, \\ Slovenia \\ E-mail: dusan.repovs@guest.arnes.si
}

received 13 Dec 2007, revised 26 May 2008, 12 Jan 2010, accepted 15 Jan 2010.

We prove that for each partition of the Lobachevsky plane into finitely many Borel pieces one of the cells of the partition contains an unbounded centrally symmetric subset.

Keywords: Partition, central symmetry, monochromatic set, Borel piece, Lobachevsky plane, Poincaré model,Borel $k$-partition, coloring

\section{Introduction}

It follows from $\left[\overline{\mathrm{B}_{1}}\right]$ (see also $\left[\mathrm{BP}_{1}\right.$, Theorem 1]) that for each partition of the $n$-dimensional space $\mathbb{R}^{n}$ into $n$ pieces one of the pieces contains an unbounded centrally symmetric subset. On the other hand, $\mathbb{R}^{n}$ admits a partition $\mathbb{R}^{n}=B_{0} \cup \cdots \cup B_{n}$ into $(n+1)$ Borel pieces containing no unbounded centrally symmetric subset. For $n=2$ such a partition is drawn at the picture:

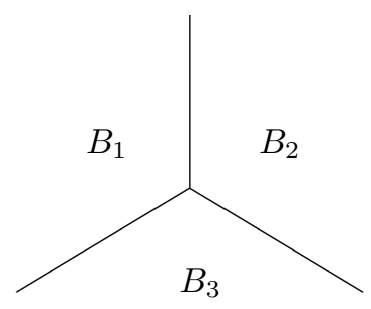


Taking the same partition of the Lobachevsky plane $H^{2}$, we can see that each piece $B_{i}$ does contain an unbounded centrally symmetric subset (for such a set just take any hyperbolic line lying in $B_{i}$ ).

We call a subset $S$ of the hyperbolic plane $H^{2}$ centrally symmetric or else symmetric with respect to a point $c \in H^{2}$ if $S=f_{c}(S)$ where $f_{c}: H^{2} \rightarrow H^{2}$ is the involutive isometry of $H^{2}$ assigning to each point $x \in H^{2}$ the unique point $y \in H^{2}$ such that $c$ is the midpoint of the segment $[x, y]$. The map $f_{c}$ is called the central symmetry of $H^{2}$ with respect to the point $c$.

By a partition of a set $X$ we understand a decomposition $X=B_{1} \cup \cdots \cup B_{n}$ of $X$ into pairwise disjoint subsets called the pieces of the partition.

The following theorem shows that the Lobachevsky plane differs dramatically from the Euclidean plane from the Ramsey point of view.

Theorem 1.1 For any partition $H^{2}=B_{1} \cup \cdots \cup B_{m}$ of the Lobachevsky plane into finitely many Borel pieces one of the pieces contains an unbounded centrally symmetric subset.

\section{Proof of Theorem 1.1}

We shall prove a bit more: given a partition $H^{2}=B_{1} \cup \cdots \cup B_{m}$ of the Lobachevsky plane into $m$ Borel pieces we shall find $i \leq m$ and an unbounded subset $S \subset B_{i}$ symmetric with respect to some point $c$ in an arbitrarily small neighborhood of some finite set $F \subset H^{2}$ depending only on $m$.

To define this set $F$ it will be convenient to work in the Poincaré model of the Lobachevsky plane $H^{2}$. In this model the hyperbolic plane $H^{2}$ is identified with the unit disk $\mathbb{D}=\{z \in \mathbb{C}:|z|<1\}$ on the complex plane and hyperbolic lines are just segments of circles orthogonal to the boundary of $\mathbb{D}$. Let $\overline{\mathbb{D}}=\{z \in \mathbb{C}:|z| \leq 1\}$ be the hyperbolic plane $\mathbb{D}$ with attached ideal line. For a real number $R>0$ the set $\mathbb{D}_{R}=\{z \in \mathbb{C}:|z| \leq 1-1 / R\}$ can be thought as a hyperbolic disk of increasing radius as $R$ tends to $\infty$.

On the boundary of the unit disk $\mathbb{D}$ consider the $(m+1)$-element set

$$
A=\left\{z \in \mathbb{C}: z^{m+1}=1\right\}
$$

For two distinct points $x, y \in A$ let $[x \mid y] \in \mathbb{D}$ denote the midpoint of the arc in $\overline{\mathbb{D}}$ that connects the points $x, y$ and lies on a hyperbolic line in $H^{2}=\mathbb{D}$. Then $F=\{[x \mid y]: x, y \in A, x \neq y\}$ is a finite subset of cardinality $|F| \leq m(m+1) / 2$ in the unit disk $\mathbb{D}$.

For $m=3$ the set $A$ consists of four points $a_{1}=1, a_{2}=i, a_{3}=-1$ and $a_{4}=-i$ while $F$ consists of five points $\left[a_{1} \mid a_{2}\right],\left[a_{2} \mid a_{3}\right],\left[a_{3} \mid a_{4}\right],\left[a_{4} \mid a_{1}\right],\left[a_{1} \mid a_{3}\right]=\left[a_{2} \mid a_{4}\right]$ as shown at the following picture: 


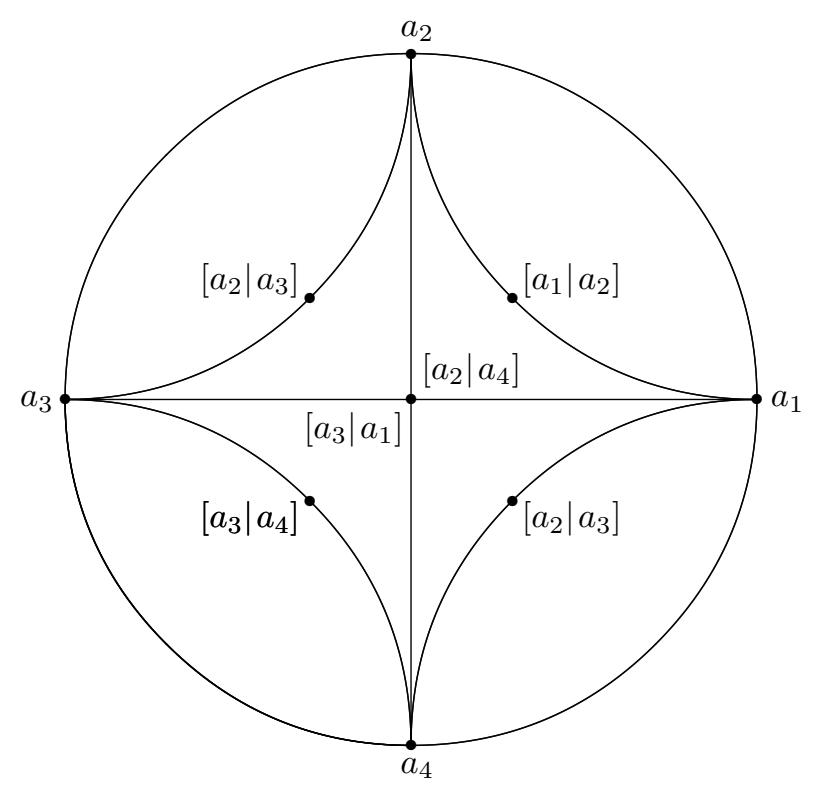

We claim that for any open neighborhood $W$ of $F$ in $\mathbb{C}$ one of the pieces of a partition $H^{2}=B_{1} \cup \cdots \cup$ $B_{m}$ contains an unbounded subset symmetric with respect to some point $c \in W$. To derive a contradiction we assume the converse: for every point $c \in W$ and every $i \leq m$ the set $B_{i} \cap f_{c}\left(B_{i}\right)$ is bounded in $H^{2}$.

For every $n \in \mathbb{N}$ consider the set

$$
C_{n}=\left\{c \in W: \bigcup_{i=1}^{m} B_{i} \cap f_{c}\left(B_{i}\right) \subset \mathbb{D}_{n}\right\} .
$$

We claim that $C_{n}$ is a coanalytic subset of $W$, that is, the complement $W \backslash C_{n}$ is analytic, which in its turn means that $W \backslash C_{n}$ is the continuous image of a Polish space. Observe that

$$
W \backslash C_{n}=\left\{c \in W: \text { there are } i \leq m \text { and } x \in B_{i} \cap f_{c}\left(B_{i}\right) \backslash \mathbb{D}_{n}\right\}=\operatorname{pr}_{2}(E),
$$

where $\operatorname{pr}_{2}: \mathbb{D} \times \mathbb{D} \rightarrow \mathbb{D}$ is the projection on the second factor and

$$
E=\bigcup_{i=1}^{m}\left\{(x, c) \in \mathbb{D} \times W: x \in B_{i} \cap f_{c}\left(B_{i}\right) \backslash \mathbb{D}_{n}\right\}
$$

is a Borel subset of $\mathbb{D} \times W$. Being a Borel subset of the Polish space $\mathbb{D} \times W$, the space $E$ is analytic and so is its continuous image $\operatorname{pr}_{2}(E)=W \backslash C_{n}$. Then $C_{n}$ is coanalytic and hence has the Baire property [Ke, 21.6], which means that $C_{n}$ coincides with an open subset $U_{n}$ of $W$ modulo some meager set. The latter means that the symmetric difference $U_{n} \triangle C_{n}$ is meager (i.e., is of the first Baire category) in $W$. Replacing $U_{n}$ by the interior of the closure $\bar{U}_{n}$ of $U_{n}$ in $W$, if necessary, we may additionally assume that $U_{n}$ is regular open, that is, $U_{n}$ coincides with the interior of its closure in $W$. 
We claim that $C_{n} \subset C_{n+1}$ implies $U_{n} \subset U_{n+1}$. First we check that

$$
U_{n} \backslash U_{n+1} \subset\left(U_{n} \triangle C_{n}\right) \cup\left(U_{n+1} \triangle C_{n+1}\right)
$$

is meager. Indeed, for every $x \in U_{n} \backslash U_{n+1}$ we get $x \in U_{n} \backslash C_{n} \subset U_{n} \triangle C_{n}$ if $x \notin C_{n}$ and $x \in$ $C_{n+1} \backslash U_{n+1} \subset U_{n+1} \triangle C_{n+1}$ if $x \in C_{n} \subset C_{n+1}$. Therefore, the set $U_{n} \backslash U_{n+1}$ is meager, which implies $U_{n} \subset \bar{U}_{n+1}$ and hence $U_{n} \subset U_{n+1}$ because the set $U_{n+1}$ is regular open.

Let $U=\bigcup_{n=1}^{\infty} U_{n}$ and $M=\bigcup_{n=1}^{\infty} U_{n} \triangle C_{n}$. Taking into account that $W=\bigcup_{n=1}^{\infty} C_{n}$, we conclude that

$$
W \backslash U=\bigcup_{n=1}^{\infty} C_{n} \backslash \bigcup_{n=1}^{\infty} U_{n} \subset \bigcup_{n=1}^{\infty} C_{n} \backslash U_{n} \subset \bigcup_{n=1}^{\infty} C_{n} \triangle U_{n}=M
$$

which implies that the open set $U$ has meager complement and thus is dense in $W$.

We claim that $F \subset h^{-1}(U)$ for some isometry $h$ of the hyperbolic plane $H^{2}=\mathbb{D}$.

For this consider the natural action

$$
\mu: \operatorname{Iso}\left(H^{2}\right) \times \mathbb{D} \rightarrow \mathbb{D}, \quad \mu:(h, x) \mapsto h(x)
$$

of the isometry group Iso $\left(H^{2}\right)$ of the hyperbolic plane $H^{2}=\mathbb{D}$. It is easy to see that for every $x \in \mathbb{D}$ the map $\mu_{x}: \operatorname{Iso}\left(H^{2}\right) \rightarrow \mathbb{D}, \mu_{x}: h \mapsto h(x)$, is continuous and open (with respect to the compact-open topology on $\operatorname{Iso}\left(H^{2}\right)$ ). It follows that the set

$$
\bigcap_{x \in F} \mu_{x}^{-1}(W)=\left\{h \in \operatorname{Iso}\left(H^{2}\right): h(F) \subset W\right\}
$$

is an open neighborhood of the neutral element of the group $\operatorname{Iso}\left(H^{2}\right)$.

Taking into account that $U$ is open and dense in $W$, and that for every $x \in F$ the map $\mu_{x}: \operatorname{Iso}\left(H^{2}\right) \rightarrow$ $\mathbb{D}$ is open, we conclude that the preimage $\mu_{x}^{-1}(U)$ is open and dense in $\mu_{x}^{-1}(W) \subset \operatorname{Iso}\left(H^{2}\right)$. Then the intersection $\bigcap_{x \in F} \mu_{x}^{-1}(U)$, being an open dense subset of $\bigcap_{x \in F} \mu_{x}^{-1}(W)$, is not empty and hence contains some isometry $h$ having the desired property: $F \subset h^{-1}(U)$. Since $F$ is finite, there is $n \in \mathbb{N}$ with $F \subset h^{-1}\left(U_{n}\right)$. For a complex number $r \in \mathbb{D}$ consider the set $r A=\{r z: z \in A\} \subset \mathbb{D}$ and let

$$
F_{r}=\{[x \mid y]: x, y \in r A, x \neq y\} \subset \mathbb{D},
$$

where $[x \mid y]$ stands for the midpoint of the hyperbolic segment connecting $x$ and $y$ in $H^{2}$. It can be shown that for any distinct points $x, y \in A$ the midpoint $[r x \mid r y]$ tends to the midpoint $[x \mid y] \in F$ as $r$ tends to 1 . Such a continuity yields a neighborhood $O_{1}$ of 1 such that $F_{r} \subset h^{-1}\left(U_{n}\right)$ for all $r \in O_{1} \cap \mathbb{D}$.

It is clear that for any points $x, y \in A$ the map

$$
f_{x, y}: \mathbb{D} \rightarrow \mathbb{D}, f_{x, y}: r \mapsto[r x \mid r y]
$$

is open and continuous. Consequently, the preimage $f_{x, y}^{-1}\left(h^{-1}(M)\right)$ is a meager subset of $\mathbb{D}$ and so is the union $M^{\prime}=\bigcup_{x, y \in A} f_{x, y}^{-1}\left(h^{-1}(M)\right)$. So, we can find a non-zero point $r \in O_{1} \backslash M^{\prime}$ so close to 1 that the set $r A$ is disjoint with the hyperbolic disk $h^{-1}\left(\mathbb{D}_{n}\right)$ (observe that for a complex number $r$ close to 1 the set $r A$ is close to the set $A$ lying in the boundary circle of $\mathbb{D}$ and thus $r A$ can be made disjoint with the compact subset $h^{-1}\left(\mathbb{D}_{n}\right)$ of $\left.\mathbb{D}\right)$. For this point $r$ we shall get $F_{r} \cap h^{-1}(M)=\emptyset$. 
The set $r A$ consists of $m+1$ points. Consequently, some cell $h^{-1}\left(B_{i}\right)$ of the partition $\mathbb{D}=h^{-1}\left(B_{1}\right) \cup$ $\cdots \cup h^{-1}\left(B_{m}\right)$ contains two distinct points $r x$, ry of $r A$. Those points are symmetric with respect to the point

$$
[r x \mid r y] \in F_{r} \subset h^{-1}\left(U_{n}\right) \backslash h^{-1}(M) .
$$

Then the images $a=h(r x)$ and $b=h(r y)$ belong to $B_{i}$ and are symmetric with respect to the point $c=h([r x \mid r y]) \in U_{n} \backslash M \subset C_{n}$. It follows from the definition of $C_{n}$ that $\{a, b\} \subset B_{i} \cap f_{c}\left(B_{i}\right) \subset \mathbb{D}_{n}$, which is not the case because $r x, r y \notin h^{-1}\left(\mathbb{D}_{n}\right)$.

\section{Concerning partitions of $H^{2}$}

We do not know if Theorem 1.1 is true for any finite (not necessarily Borel) partition of the Lobachevsky plane $H^{2}$. For partitions of $H^{2}$ into two pieces, the Borel assumption is superfluous.

Theorem 3.1 There is a subset $T \subset H^{2}$ of cardinality $|T|=3$ such that for any partition $H^{2}=A_{1} \cup A_{2}$ of $\mathrm{H}^{2}$ into two pieces either $A_{1}$ or $A_{2}$ contains an unbounded subset, symmetric with respect to some point $c \in T$.

Proof: Lemma 3.2 below allows us to find an equilateral triangle $\triangle c_{0} c_{1} c_{2}$ on the Lobachevsky plane $H^{2}$ such that the composition $f_{c_{2}} \circ f_{c_{1}} \circ f_{c_{0}}$ of the symmetries with respect to the points $c_{0}, c_{1}, c_{2}$ coincides with the rotation by the angle $2 \pi / 3$ about some point $o \in H^{2}$. Consequently $\left(f_{c_{2}} \circ f_{c_{1}} \circ f_{c_{0}}\right)^{3}$ is the identity isometry of $H^{2}$.

We claim that for any partition $H^{2}=A_{1} \sqcup A_{2}$ of the Lobachevsky plane into two pieces one of the pieces contains an unbounded subset symmetric with respect to some point in the triangle $T=$ $\left\{c_{0}, c_{1}, c_{2}\right\}$. Assuming the converse, we conclude that the set

$$
B=\bigcup_{c \in T} \bigcup_{i=1}^{2} A_{i} \cap f_{c}\left(A_{i}\right)
$$

is bounded. It follows that two points $x, y \in H^{2} \backslash B$, symmetric with respect to a center $c \in T$ cannot belong to the same cell $A_{i}$ of the partition.

Let $B_{0}=B$ and $B_{i+1}=B_{i} \cup \bigcup_{j=0}^{2} f_{c_{j}}^{-1}\left(B_{i}\right)$ for $i \geq 0$. By induction it can be shown that each set $B_{i}$, $i \geq 0$, is bounded in $H^{2}$.

Fix any point $x_{0} \in H^{2} \backslash B_{9}$ and consider the sequence of points $x_{1}, \ldots x_{9}$ defined by the recursive formula: $x_{i+1}=f_{c_{i \text { mod } 3}}\left(x_{i}\right)$. It follows that

$$
x_{9}=\left(f_{c_{2}} \circ f_{c_{1}} \circ f_{c_{0}}\right)^{3}\left(x_{0}\right)=x_{0} .
$$

We claim that for every $i \leq 9$ the point $x_{i}$ does no belong to the set $B$. Assuming by contradiction that $x_{i} \in B$, we would conclude that $x_{i-1} \in \bigcup_{j=0}^{2} f_{c_{j}}^{-1}(B) \subset B_{1}$. Continuing by induction, for every $k \leq i$ we would get $x_{i-k} \in B_{k}$. In particular, $x_{0} \in B_{i} \subset B_{9}$, which contradicts the choice of $x_{0}$.

The point $x_{0}$ belongs either to $A_{1}$ or to $A_{2}$. We loose no generality assuming that $x_{0} \in A_{2}$. Since the points $x_{0}, x_{1} \notin B$ are symmetric with respect to $c_{0}$ and $x_{0} \in A_{2}$, we get that $x_{1} \in H^{2} \backslash A_{2}=A_{1}$. By the same reason $x_{1}, x_{2}$ cannot simultaneously belong to $A_{1}$ and hence $x_{2} \in A_{2}$. Continuing in this fashion we conclude that $x_{i}$ belongs to $A_{1}$ for odd $i$ and to $A_{2}$ for even $i$. In particular, $x_{9} \in A_{1}$, which is not possible because $x_{9}=x_{0} \in A_{2}$. 
Lemma 3.2 There is an equilateral triangle $\triangle A B C$ on the Lobachevsky plane such that the composition $f_{C} \circ f_{B} \circ f_{A}$ of the symmetries with respect to the points $A, B, C$ coincides with the rotation by the angle $2 \pi / 3$ about some point $O$.

Proof: For a positive real number $t$ consider an equilateral triangle $\triangle A B C$ with side $t$ the on the Lobachevsky plane. Let $M$ be the midpoint of the side $A B$ and $l$ be the line through $C$ that is orthogonal to the line $C M$. Consider also the line $p$ that is orthogonal to the line $A B$ and passes through the point $P$ such that $A$ is the midpoint between $P$ and $M$. Observe that $|P M|=|A B|=t$ and for sufficiently small $t$ the lines $p$ and $l$ intersect at some point $O$.

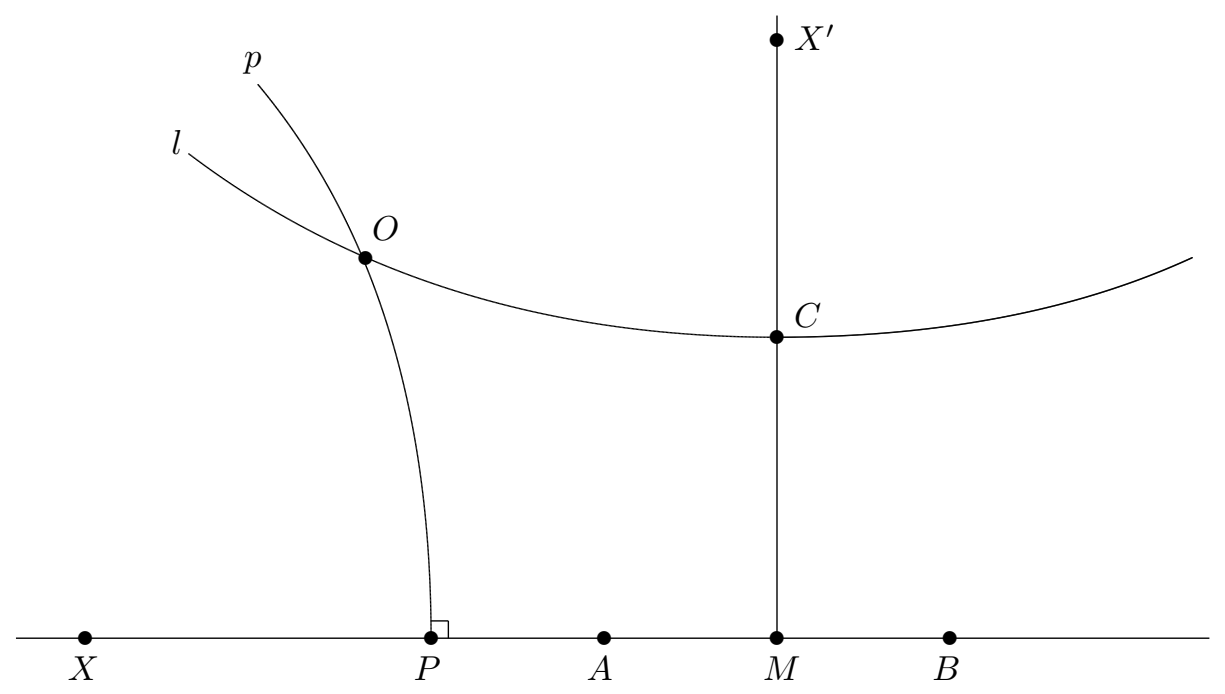

It is easy to see that the composition $f_{B} \circ f_{A}$ is the shift along the line $A B$ by the distance $2 t$ and hence the image $f_{B} \circ f_{A}(O)$ of the point $O$ is the point symmetric to $O$ with respect to the point $C$. Consequently, $f_{C} \circ f_{B} \circ f_{A}(O)=O$, which means that the isometry $f_{C} \circ f_{B} \circ f_{A}$ is a rotation of the Lobachevsky plane about the point $O$ by some angle $\varphi_{t}$.

To estimate this angle, consider the point $X$ such that $P$ is the midpoint between $X$ and $M$. Then $|X M|=2 t$ and consequently, $f_{B} \circ f_{A}(X)=M$ while $X^{\prime}=f_{C} \circ f_{B} \circ f_{A}=f_{C}(M)$ is the point on the line $C M$ such that $C$ is the midpoint between $X^{\prime}$ and $M$. It follows that $\left|X^{\prime} X\right| \leq|X M|+\left|M X^{\prime}\right|<$ $2 t+2 t=4 t$.

Observe that for small $t$ the point $X^{\prime}$ is near to the point, symmetric to $X$ with respect to $O$, which means that the angle $\varphi_{t}=\angle X O X^{\prime}$ is close to $\pi$ for $t$ close to zero. On the other hand, for very large $t$ the lines $p$ and $l$ on the Lobachevsky plane do not intersect. So we can consider the smallest upper bound $t_{0}$ of numbers $t$ for which the lines $l$ and $p$ meet. For values $t<t_{0}$ near to $t_{0}$ the point $O$ tends to infinity as $t$ tends to $t_{0}$. Since the length of the side $X X^{\prime}$ of the triangle $\triangle X O X^{\prime}$ is bounded by $4 t_{0}$ the angle $\varphi_{t}=\angle X O X^{\prime}$ tends to zero as $O$ tends to infinity. Since the angle $\varphi_{t}$ depends continuously on $t$ and decreases from $\pi$ to zero as $t$ increases from zero to $t_{0}$, there is a value $t$ such that $\varphi_{t}=2 \pi / 3$. For such $t$ the composition $f_{C} \circ f_{B} \circ f_{A}$ is the rotation around $O$ on the angle $2 \pi / 3$. 


\section{Some comments and open problems}

In contrast with Theorem 1.1. Theorem 3.1 is true for the Euclidean plane $E^{2}$ even in a stronger form: for any subset $C \subset E^{2}$ not lying on a line and any partition $E^{2}=A_{1} \cup A_{2}$ one of the cells of the partition contains an unbounded subset symmetric with respect to some center $c \in C$, see $\left[\overline{\mathrm{B}_{2}}\right]$.

Having in mind this result let us call a subset $C$ of a Lobachevsky or Euclidean space $X$ central for (Borel) k-partitions if for any partition $X=A_{1} \cup \cdots \cup A_{k}$ of $X$ into $k$ (Borel) pieces one of the pieces contains an unbounded monochromatic subset $S \subset X$, symmetric with respect to some point $c \in C$. By $c_{k}(X)$ (resp. $\left.c_{k}^{B}(X)\right)$ we shall denote the smallest size of a subset $C \subset X$, central for (Borel) $k$-partitions of $X$. If no such set $C$ exists, then we put $c_{k}(X)=\infty$ (resp. $c_{k}^{B}(X)=\infty$ ) where $\infty$ is assumed to be greater than any cardinal number. It follows from the definition that $c_{k}^{B}(X) \leq c_{k}(X)$.

We have a lot of information about the numbers $c_{k}^{B}\left(E^{n}\right)$ and $c_{k}\left(E^{n}\right)$ for Euclidean spaces $E^{n}$, see $\left[\mathrm{B}_{2}\right]$. In particular, we known that

1. $c_{2}\left(E^{n}\right)=c_{2}^{B}\left(E^{n}\right)=3$ for all $n \geq 2$;

2. $c_{3}\left(E^{3}\right)=c_{3}^{B}\left(E^{3}\right)=6$;

3. $12 \leq c_{4}^{B}\left(E^{4}\right) \leq c_{4}\left(E^{4}\right) \leq 14$;

4. $n(n+1) / 2 \leq c_{n}^{B}\left(E^{n}\right) \leq c_{n}\left(E^{n}\right) \leq 2^{n}-2$ for every $n \geq 3$.

Much less is known about the numbers $c_{k}^{B}\left(H^{n}\right)$ and $c_{k}^{B}\left(H^{n}\right)$ in the hyperbolic case. Theorem 3.1 yields the upper bound $c_{2}\left(H^{2}\right) \leq 3$. In fact, 3 is the exact value of $c_{2}\left(H^{n}\right)$ for all $n \geq 2$.

Proposition 4.1 $c_{2}^{B}\left(H^{n}\right)=c_{2}\left(H^{n}\right)=3$ for all $n \geq 2$.

Proof: The upper bound $c_{2}\left(H^{n}\right) \leq c_{2}\left(H^{2}\right) \leq 3$ follows from Theorem 3.1 The lower bound $3 \leq$ $c_{2}^{B}\left(H^{n}\right)$ will follow as soon as for any two points $c_{1}, c_{2} \in H^{n}$ we construct a partition $H^{n}=A_{1} \cup A_{2}$ in two Borel pieces containing no unbounded set, symmetric with respect to a point $c_{i}$. To construct such a partition, consider the line $l$ containing the points $c_{1}, c_{2}$ and decompose $l$ into two half-lines $l=l_{1} \sqcup l_{2}$. Next, let $H$ be an $(n-1)$-hyperplane in $H^{n}$, orthogonal to the line $l$. Let $S$ be the unit sphere in $H$ centered at the intersection point of $l$ and $H$. Let $S=B_{1} \cup B_{2}$ be a partition of $S$ into two Borel pieces such that no antipodal points of $S$ lie in the same cell of the partition. For each point $x \in H^{n} \backslash l$ consider the hyperbolic plane $P_{x}$ containing the points $x, c_{1}, c_{2}$. The complement $P_{x} \backslash l$ decomposes into two half-planes $P_{x}^{+} \cup P_{x}^{-}$where $P_{x}^{+}$is the half-plane containing the point $x$. The plane $P_{x}$ intersects the hyperplane $H$ by a hyperbolic line containing two points of the sphere $S$. Finally put

$$
A_{i}=l_{i} \cup\left\{x \in H^{2} \backslash l: P_{x}^{+} \cap B_{i} \neq \emptyset\right\}
$$

for $i \in\{1,2\}$. It is easy to check that $A_{1} \sqcup A_{2}=H^{n}$ is the desired partition of the hyperbolic space into two Borel pieces none of which contains an unbounded subset symmetric with respect to one of the points $c_{1}, c_{2}$.

The preceding proposition implies that the cardinal numbers $c_{2}\left(H^{n}\right)$ are finite.

Problem 4.2 For which numbers $k, n$ are the cardinal numbers $c_{k}\left(H^{n}\right)$ and $c_{k}^{B}\left(H^{n}\right)$ finite? Is it true for all $k \leq n$ ? 
Except for the equality $c_{2}\left(E^{n}\right)=3$, we have no information on the numbers $c_{k}\left(E^{n}\right)$ with $k<n$.

Problem 4.3 Calculate (or at least evaluate) the numbers $c_{k}\left(E^{n}\right)$ and $c_{k}\left(H^{n}\right)$ for $2<k<n$.

In all the cases where we know the exact values of the numbers $c_{k}\left(E^{n}\right)$ and $c_{k}^{B}\left(E^{n}\right)$ we see that those numbers are equal.

Problem 4.4 Are the numbers $c_{k}\left(E^{n}\right)$ and $c_{k}^{B}\left(E^{n}\right)$ (resp. $c_{k}\left(H^{n}\right)$ and $c_{k}^{B}\left(H^{n}\right)$ ) equal for all $k, n$ ?

Having in mind that each subset not lying on a line is central for 2-partitions of the Euclidean plane, we may ask about the same property of the Lobachevsky plane.

Problem 4.5 Is any subset $C \subset H^{2}$ not lying on a line central for (Borel) 2-partitions of the Lobachevsky plane $H^{2}$ ?

Finally, let us ask about the numbers $c_{k}^{B}\left(H^{2}\right)$ and $c_{k}\left(H^{2}\right)$. Observe that Theorem 1.1 guarantees that $c_{k}^{B}\left(H^{2}\right) \leq \mathfrak{c}$ for all $k \in \mathbb{N}$. Inspecting the proof we can see that this upper bound can be improved to $c_{k}^{B}\left(H^{2}\right) \leq \operatorname{non}(\mathcal{M})$ where $\operatorname{non}(\mathcal{M})$ is the smallest cardinality of a non-meager subset of the real line. It is clear that $\aleph_{1} \leq \operatorname{non}(\mathcal{M}) \leq \mathfrak{c}$. The exact location of the cardinal non $(M)$ on the interval $\left[\aleph_{1}, \mathfrak{c}\right]$ depends on axioms of Set Theory, see [B]]. In particular, the inequality $\aleph_{1}=\operatorname{non}(\mathcal{M})<\mathfrak{c}$ is consistent with ZFC.

Problem 4.6 Is the inequality $c_{k}^{B}\left(H^{2}\right) \leq \aleph_{1}$ provable in ZFC? Are the cardinals $c_{k}^{B}\left(H^{2}\right)$ countable? finite?

The last problem asks if $H^{2}$ contains a countable (or finite) central set for Borel $k$-partitions of the Lobachevsky plane. Inspecting the proof of Theorem 1.1 we can see that it gives an "approximate" answer to this problem:

Proposition 4.7 For any $k \in \mathbb{N}$ there is a finite subset $C \subset H^{2}$ of cardinality $|C| \leq k(k+1) / 2$ such that for any partition $H^{2}=B_{1} \cup \cdots \cup B_{k}$ of $H^{2}$ into $k$ Borel pieces and for any open neighborhood $O(C) \subset H^{2}$ of $C$ one of the pieces $B_{i}$ contains an unbounded subset $S \subset B_{i}$ symmetric with respect to some point $c \in O(C)$.

Remark 4.8 For further results and open problems related to symmetry and colorings see the surveys $\left[B P_{2}\right],[B V V]$ and the list of problems [BBGRZ $\left.\S 4\right]$.

\section{Acknowledgements}

This reseach was supported the Slovenian Research Agency grants P1-0292-0101, J1-2057-0101 and BIUA/09-10-002. The authors express their sincere thanks to Christian Krattenthaler for several comments and suggestions. 


\section{References}

[B $\left.\mathrm{B}_{1}\right] \quad \mathrm{T}$. Banakh, Solutions of certain problems of I.V.Protasov from the combinatorics of colorings, U Sviti Mat. 3:1 (1997), 8-11 (in Ukrainian).

$\left[\mathrm{B}_{2}\right] \quad$ T. Banakh, On a cardinal group invariant related to partition of abelian groups, Mat. Zametki. 64:3 (1998), 341-350.

[BBGRZ] T. Banakh, B. Bokalo, I. Guran, T. Radul, M. Zarichnyi, Problems from the Lviv topological seminar, in: Open Problems in Topology, II (E.Pearl, Ed.), Elsevier North-Holland, Amsterdam, 2007, pp. 655-667.

[BP $\left.{ }_{1}\right] \quad$ T. Banakh, I. Protasov, Asymmetric partitions of abelian groups, Mat. Zametki, 66 (1999), 10-19.

$\left[\mathrm{BP}_{2}\right] \quad$ T. Banakh, I. Protasov, Symmetry and colorings: some results and open problems, Izv. Gomel Univ. Voprosy Algebry. 4 (2001), 5-16 (arXiv:0901.3356).

[BVV] T. Banakh, O. Verbitsky, Ya. Vorobets, A Ramsey treatment of symmetry, Electron. J. Combin. 7 (2000), R52, 25 pp.

[B1] A. Blass, Combinatorial cardinal characteristics of the continuum, in: Handbook of Set Theory (M. Foreman, M. Magidor, A. Kanamoried, eds.), Springer-Verlag, Berlin, 2007.

[Ke] A. Kechris, Classical Descriptive Set Theory, Springer-Verlag, Berlin, 1995. 